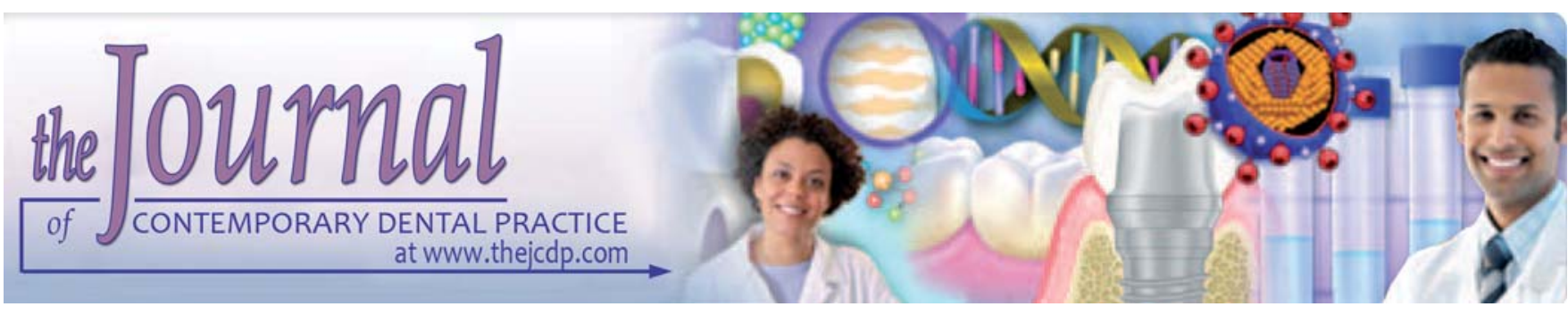

\title{
Single Visit Replacement of Maxillary Canine using Fiber-reinforced Composite Resin
}

\author{
Sufyan Garoushi, Lippo Lassila, Pekka K Vallittu
}

\begin{abstract}
Missing a canine is of serious concern in social life of a patient in most of societies. While conventional fixed partial dentures and implant-supported restorations may often be the treatment of choice, fiber-reinforced composite (FRC) resins offer a conservative, fast and cost-effective alternative for single and multiple teeth replacement. This clinical report presents two cases where FRC technology was successfully used to restore canine edentulous area in terms of esthetic-cosmetic values and functionality.
\end{abstract}

Keywords: Case report, Composite resin, Fiber-reinforced composite, Resin-bonded fixed partial dentures.

How to cite this article: Garoushi S, Lassila L, Vallittu PK. Single Visit Replacement of Maxillary Canine using Fiber-reinforced Composite Resin. J Contemp Dent Pract 2012;13(1):125-129.

Source of support: Nil

Conflict of interest: None declared

\section{INTRODUCTION}

Loss of anterior teeth is a common form of injury, particularly in children and adolescents. On the other hand side, elderly people who are retaining their teeth for longer period of time, have often advanced caries or periodontal diseases which may lead to extraction of teeth. Patients with lost anterior teeth require immediate attention for restoration of esthetic-cosmetic and functional reasons. With increased patient demand for tissue maintenance and esthetic, as well as to reduce treatment costs, causes clinician to seek materials and techniques that enable minimally invasive and chairside (direct) fabrication on teeth replacement with fixed partial dentures (FPD).

Over the last few years, the development of fiberreinforced composite (FRC) has offered the dental profession the possibility of fabricating resin-bonded, esthetically good and metal-free tooth restorations for single and multiple teeth replacement. FRC-FPD could be an alternative to metal frame resin-bonded FPD, and also to full coverage crown retained FPD and implant supported crowns. ${ }^{1,2}$ FRC, made of glass fibers is the only existing material cosmetically acceptable material, which can be processed in mouth to the shape of a framework of a bridge, simultaneously adhere to the remaining tooth substance, and reach the adequate strength in terms of biting function of human. Many studies have focused on improvement of FRC FPD's strength. ${ }^{3,4}$ The most accepted concept to fabricate FRC FPDs is based on the use of continuous unidirectional glass (bundle) fibers in dimethacrylate-polymethylmethacrylate resin matrix as a substructure for the FPD. ${ }^{5}$ With the FRC FPDs, there are two approaches on the use of the fibers: One is based on conventional tooth preparation and laboratory-made restorations while the other is based on using the fibers in minimally invasive restoration (conservative) by direct or indirect fabrication. FRC systems enable the use of different retainer elements even in the same FPD (hybrid type). ${ }^{2}$ For example, it is possible to create space for the occlusal support of the FRC framework by removing old filling, or to make completely surface-retained restorations when clinical conditions allow correct designing of the FRC framework. In the dental literature, there are presently on limited number of clinical studies on the fiberreinforced FPDs, however, based on those results, it is reasonable to expect FRC prostheses to have good longevity, especially with those made by direct technique. ${ }^{2,6,7}$

This article describes clinical cases of chairside (directly) made FRC FPDs, which was used according to the principles of minimal invasiveness.

\section{CASE REPORT}

\section{Case 1}

A 33-year-old male patient had a chief complaint of esthetics and cosmetics because of a gap of missing canine (Fig. 1). 
After discussion with the patient, it became clear that the placement of an implant for the replacement of missing canine was not possible due to high costs of the treatment. The fabrication of a conventional fixed partial denture was avoided and refused from patient in order to conserve the remaining tooth substance. Option for the conventional treatment with implants or crown retained FPDs were remained open for the future. Directly made FRC FPDs was chosen in order to provide good esthetics and cosmetics, preserve tooth substance and postpone more invasive treatments. The treatment was completed during one appointment.

There was free occlusal space on the palatal surface of lateral incisor for FRC framework to be placed. Consequently, no cavity preparation for receiving vertical support for the bridge was needed. Cotton roll for isolation was used although, the rubber dam is highly recommended. A bundle of resin impregnated glass fibers (everStick Post, Stick Tech Ltd, Turku, Finland) was cut and spread from the ends for increasing the bonding surface area (Fig. 2). The FRC framework was extended from the both buccal and palatal surfaces of premolar to palatal surface of lateral incisor (Fig. 3).

After application of acid etching (37\% phosphoric acid gel), the gel was rinsed thoroughly and gently air dried. Adhesive resins were applied according to the manufacturer's instructions (Scotchbond multipurpose adhesive, 3M ESPE, USA) to tooth surface. Flowable composite resin (Stick Flow, Stick Teck Ltd, Turku, Finland) was applied on the bonding surfaces prior placing the resin impregnated fibers (everStick). The flow composite was not light cured before fibers were pressed tightly against the tooth surface using transparent a silicone package (mold) of the fibers. The resin impregnated fibers were light cured initially through the silicone mold. The purpose of the flow composite was to seal the space between the fibers and the enamel surface. The fiber framework was polymerized two times for 40 seconds. Fiber framework was fully covered with a thin layer of flow composite resin and pontic was build up by using hybrid type particulate filler composite resin. Successful chemical bond between fiber framework and veneered composite was achieved by curing. The shade of final veneered composite resin was selected using composite shade guide and occlusion was carefully adjusted (Fig. 4). In this case canine guidance occlusion was avoided. The treatment outcome has been followed over 2 years without existence of any kind of serious problem.

\section{Case 2}

A 25-year-old male patient presented with a missing canine in the maxillary arch due to trauma since long time (Fig. 5).
The early loss of canine induced shortening of the canine crown distance, misaligned of adjacent premolar, and overeruption of lower canine. Due to high cost and long time needs for a treatment plane (orthodontic and single implant crown) replacement of missing canine was eliminated. Because patient requires orthodontic treatment to create a space for an implant crown. The fabrication of a conventional fixed partial denture was avoided in order to conserve the intact tooth substance. Directly made FRC FPDs was chosen in order to provide good esthetics and preserve tooth substance and postpone the final decision

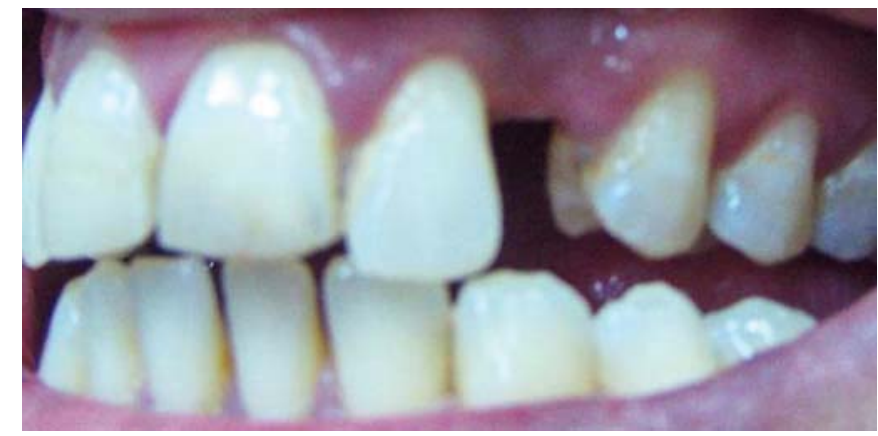

Fig. 1: Lateral view of dentition of patient no. 1 with missing canine and limited space

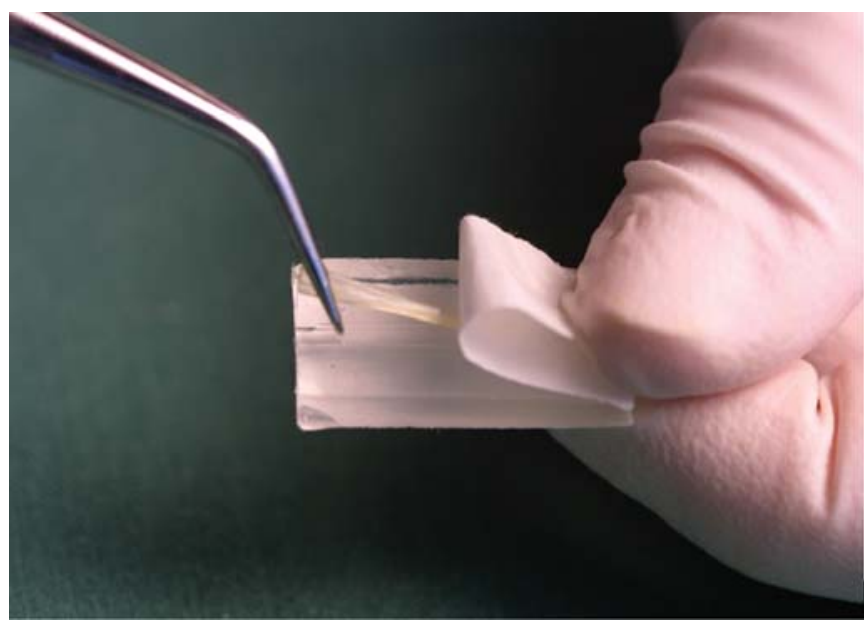

Fig. 2: Resin impregnated fiber material is taken out from the package, which can be used as mold for adapting the fibers on tooth surface

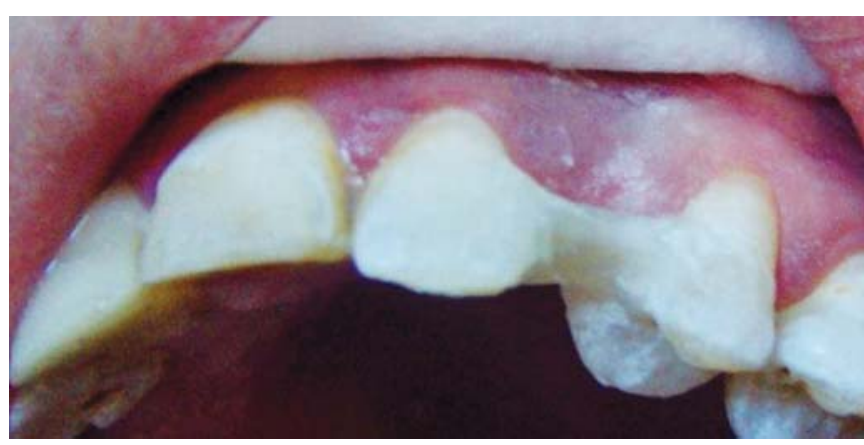

Fig. 3: Appearance of the FRC framework with a layer of flow composite between FRC and tooth 
on the prosthetic treatment. The treatment was completed during one visit appointment.

As described in the first clinical case, after etching (Fig. 6) and applying bonding agent, fiber framework was extended between the palatal surface of lateral incisor and palatal surface of rotated first premolar (Fig. 7). Fiber framework was covered with a thin layer of flow composite resin and pontic was build up by using particulate filler composite resin. The final step was the adjustment of occlusion and contouring and finishing the restoration (Fig. 8). The outcome has been monitored over 1 year with no evidence of problems.

\section{DISCUSSION}

Laboratory-made surface-retained resin-bonded prostheses made of metals are commonly supported and bonded from

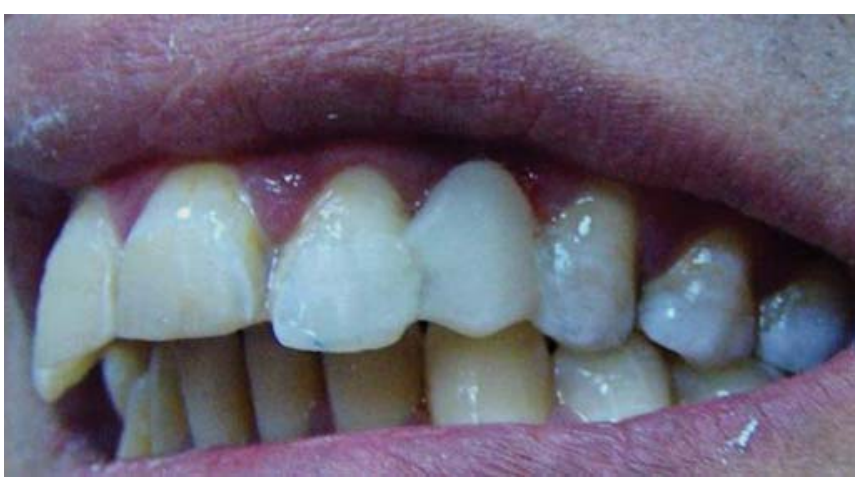

Fig. 4: Lateral view of the final restoration

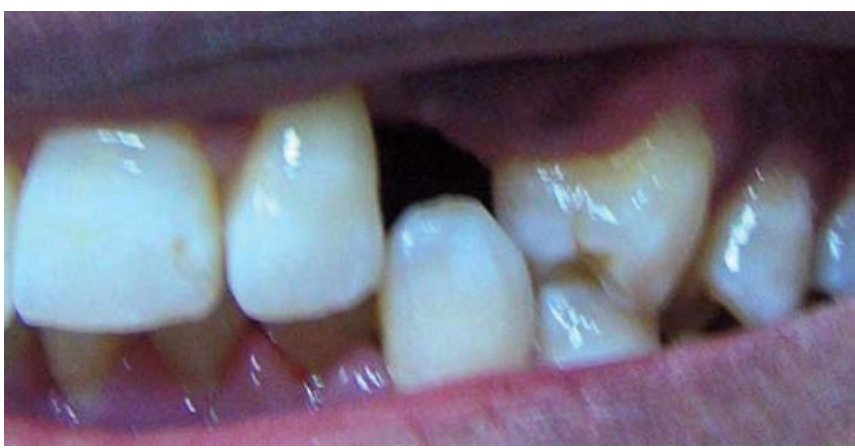

Fig. 5: Lateral view of the dentition of patient no. 2

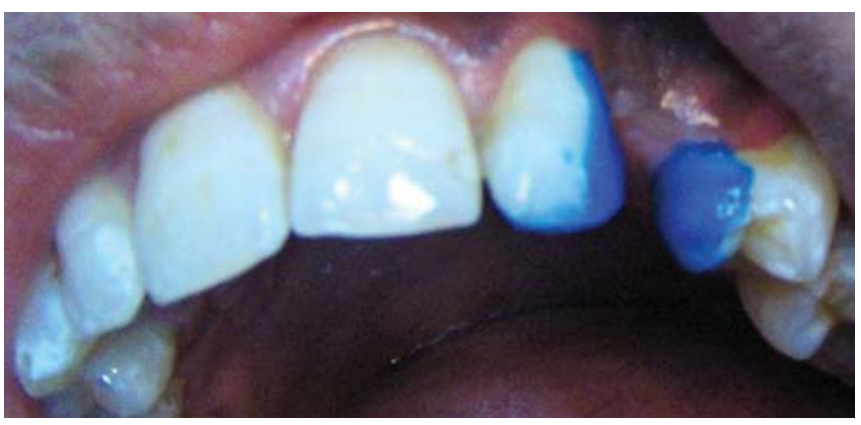

Fig. 6: Etching of teeth surfaces

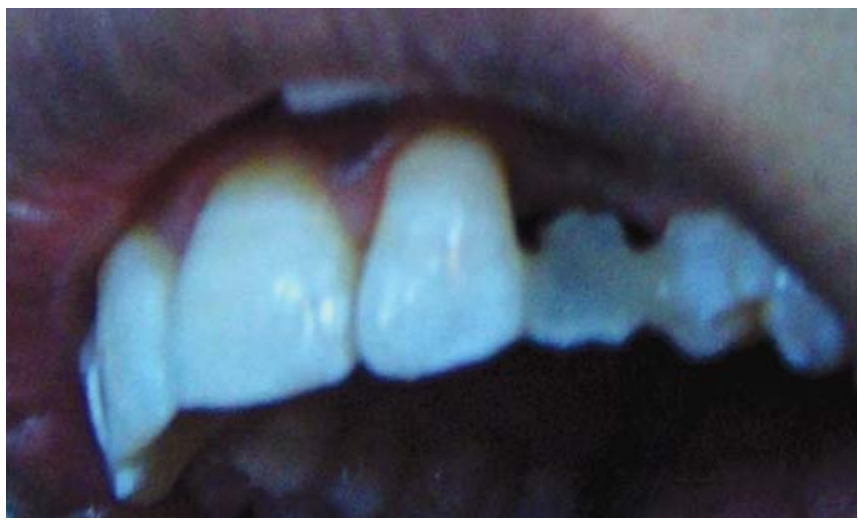

Fig. 7: Appearance of fiber framework made of everstick $C$ and $B$ fiber bundles which covered large bonding areas on adjacent teeth

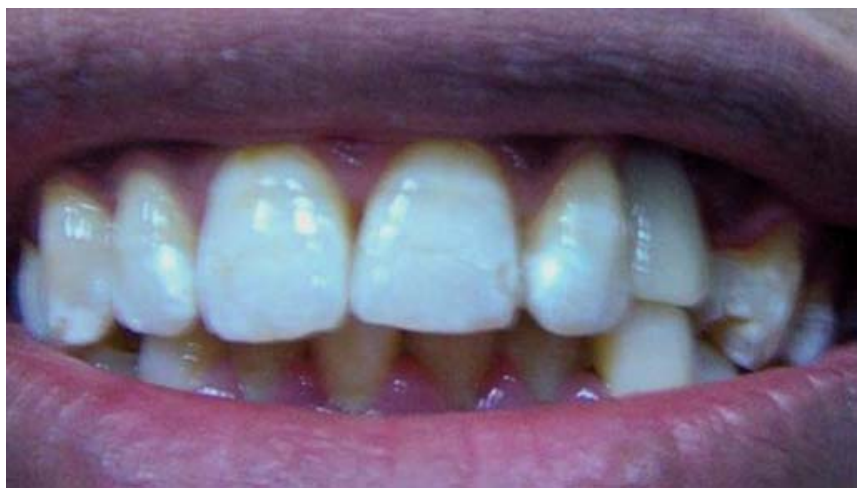

Fig. 8: Final view of the restoration having FRC framework veneered with filling composite resin

one end in order to reduce the number of debondings. In the case of surface retained FRC prostheses, the framework can be supported from both ends because of better bonding characteristics and biomechanical flexibility of the FRC framework. ${ }^{2}$ The flexibility allows abutment teeth to move without stressing the cement-framework interface in function, and loosening the prostheses. However, in the case of abutments with increased mobility, it is recommended to support also resin-bonded FRC FPD from one end only. In the cantilever designs, special care has to be taken to ensure adequate design-based rigidity of the FRC framework to resist bending forces by biting function. Adequate rigidity is obtained by increasing the cross-sectional diameter of the connector. Fibers of the framework should cover as large surface as possible on the abutments and, in the anterior area, should be placed close to the incisal edge to eliminate the dislodging forces. ${ }^{8}$

Although resin-bonded FRC FPDs are most commonly used in the anterior and premolar regions, rather than molar region, recent laboratory investigations have suggested that optimally designed FRC FPD made on nonprepared abutments can provide even higher load-bearing capacity for the FPD than conventional porcelain-fused to metal FPD can provide. ${ }^{9}$ Thus, the development of the FRC materials 
and technologies may allow alternatives also for directly made molar replacements.

The FRC framework is intended to be fully covered by veneering composite in order to obtain a polishable and tooth-colored surface. Special attention needs to be paid to the interproximal regions. If the FRC framework is not properly covered by veneering composite, the darkness of the oral cavity can be transmitted through the connectors and can cause esthetic problems. ${ }^{8}$

The composition of the polymer matrix and fiber orientation has the major role in bonding ability and durability of veneered composite to the FRC framework or resin luting cement. It has been concluded that preimpregnation of the fibers with the light-polymerizable dimethacrylate resin system containing linear polymer phases is of importance to optimize the interfacial adhesion of FRC framework to composite veneer. Using a combination of dimethacrylate monomer resin and linear polymer, which forms semi-interpenetrating polymer network (semi-IPN) after being polymerized offers better bonding site for veneered composite by means of interdiffusion bonding. ${ }^{10-12}$ Recent laboratory studies showed that bond strength of directly fabricated FRC FPD to the tooth surface is as good as particulate filler composite. $^{13}$

From clinical point of view, there is a lack of longterm clinical research of FRC prostheses. However, the longitudinal studies reported general failure rates between 5 and $16 \%$ over periods up to 4 to 5 years. ${ }^{2,7,14}$ These findings were demonstrated for prostheses with both extracoronal and intracoronal retainer designs, but only for patients who did not exhibit severe parafunctional habits. Van Heumen et al showed a survival rate of $64 \%$ after five years follow-up of 3-unit anterior FRC prostheses made with the materials and techniques used in late 1990s. ${ }^{15}$ One study reported a much higher failure rate of $40 \%$ over a 3 years period. ${ }^{16}$ The recent clinical data, with the semi-IPN resin matrix FRC FPDs made directly in patients mouth suggest high survival percentages (>96\% at 5-year), which reflects material development and learning of fabricating FRC FPDs. ${ }^{17}$ Most common failures in FRC FPDs reported in the earlier studies were delamination of veneering composite at pontic area which are normally easy to be repaired in patients mouth. The current designing principles enable to fabricate FRC FPD to eliminate known risks for technical failures.

As conclusion, the combination of filling composite veneer, adhesive system and FRC framework have introduced a new generation of metal-free direct teeth replacement. The most recent fabrication principles need to be followed to ensure clinical success of the restorations.

\section{REFERENCES}

1. Butterworth C, Ellakwa AE, Shortall A. Fiber-reinforced composites in restorative dentistry. Dent Update 2003;30: 300-06.

2. Vallittu PK, Sevelius C. Resin-bonded, glass fiber-reinforced composite fixed partial dentures: A clinical study. J Prosthet Dent 2000;84:413-18.

3. Vallittu PK. Flexural properties of acrylic resin polymers reinforced with unidirectional and woven fibers. J Prosthet Dent 1999;81:318-26.

4. Kim SH, Watts DC. Effect of glass-fiber reinforcement and water storage on fracture toughness (KIC) of polymer-based provisional crown and FPD materials. Int J Prosthodont 2004; 17:318-22.

5. Vallittu PK. Interpenetrating polymer networks (IPNs) in dental polymers and composites. J Adhes Sci Techn 2009:23; 961-72.

6. Vallittu PK. Survival rates of resin-bonded, glass fiberreinforced composite fixed partial dentures with a mean follow-up of 42 months: A pilot study. Prosthet Dent 2004;91: 241-47.

7. Monaco C, Ferrari M, Miceli GP, Scotti R. Clinical evaluation of fiber-reinforced composite inlay FPDs. Int J Prosthodont 2003;16:319-25.

8. Curtis RV, Watson TF Valittu PK. Dental biomaterials: Imaging, testing and modelling. Chapter 9, fiber-reinforced composites for dental applications. Woodhead Publishing Company 2008.

9. Dyer SR, Lassila LVJ, Alander P, Vallittu PK. Static strength of molar region direct technique glass-fiber-reinforced composite fixed partial dentures. J Oral Rehabil 2005; 32:351-57.

10. Lastumäki TM, Kallio TT, Vallittu PK. The bond strength of light-curing composite resin to finally polymerized and aged glass fibre-reinforced composite substrate. Biomaterials 2002; 23:4533-39.

11. Lastumäki TM, Lassila LV, Vallittu PK. The semi-interpenetrating polymer network matrix of fibre-reinforced composite and its effect on the surface adhesive properties. J Mater Sci Mater Med 2003;14:803-09.

12. Tezvergil-Mutluay A, Lassila LV, Vallittu PK. Microtensile bond strength of fiber-reinforced composite with semiinterpenetrating polymer matrix to dentin using various bonding systems. Dent Mater J 2008;27:821-26.

13. Tezvergil A, Lassila LV, Vallittu PK. The shear bond strength of bidirectional and random-oriented fibre-reinforced composite to tooth structure. J Dent 2005;33:509-16.

14. Freilich MA, Meiers JC, Duncan JP, Eckrote KA, Goldberg AJ. Clinical evaluation of fiber-reinforced fixed bridges. J Am Dent Assoc 2002;133:1524-34.

15. van Heumen CC, van Dijken JW, Tanner J, Pikaar R, Lassila $\mathrm{LV}$, Creugers NH, et al. Five-year survival of 3-unit fiberreinforced composite fixed partial dentures in the anterior area. Dent Mater 2009;25:820-27.

16. Bohlsen F, Kern M. Clinical outcome of glass fiber-reinforced crowns and fixed partial dentures: A three-year retrospective study. Quintessence Int 2003;34:493-96. 
17. Özcan M. Direct, inlay-retained, fiber-reinforced-composite restorations with two pontics: Five-year clinical follow-up. University Medical Center Groningen, Netherlands. IADR Toronto 2008.

\section{ABOUT THE AUTHORS}

\section{Sufyan Garoushi (Corresponding Author)}

Adjunct Professor, Department of Biomaterials Science, Institute of Dentistry and BioCity Turku Biomaterials Research Program, Turku University, Turku, Finland, e-mail: sufgar@utu.fi

\section{Lippo Lassila}

Head of Biomaterial Lab, Department of Biomaterials Science Institute of Dentistry and BioCity Turku Biomaterials Research Program, Turku University, Turku, Finland

\section{Pekka K Vallittu}

Professor, Department of Biomaterials Science, Institute of Dentistry and BioCity Turku Biomaterials Research Program, Turku University Turku, Finland 